\title{
Microstructural heterogeneities and fatigue anisotropy of forged steels
}

\author{
Etienne Pessard ${ }^{\mathrm{a}, *}$, Franck Morel $^{\mathrm{a}}$, Catherine Verdu ${ }^{\mathrm{b}}$, Laurent Flacelière ${ }^{\mathrm{c}}$, \\ Gilles Baudry ${ }^{\mathrm{C}}$ \\ ${ }^{a}$ LAMPA, Arts et Mtiers ParisTech Angers, 2 Bd du Ronceray, 49035 Angers Cedex 01 \\ ${ }^{b}$ MATEIS, INSA-Lyon, Universit de Lyon, 25 Av Jean Capelle, 69621 Villeurbanne Cedex \\ ${ }^{c}$ CREAS ASCOMETAL, BP 70045, 57301 Hagondange, France
}

\begin{abstract}
In this study, various experimental methods are employed to determine the anisotropic fatigue behavior of a $25 \mathrm{MnCrSiVB} 6$ forged steel (Metasco MC). This material has a bainitic microstructure and contains many elongated non-metallic inclusions in the rolled direction, which are grouped into clusters. Specimens with different orientations relative to the rolling direction have been extracted from a hot rolled bar and the ability of certain experimental techniques to capture the fatigue anisotropy has been tested.

Results obtained from monotonic tensile tests and Charpy impact tests show that the material has isotropic fracture strength and anisotropic ductility. The influence of the inclusion clusters is clearly demonstrated via observation of the fracture surfaces.

Concerning the fatigue behavior, results from a classical staircase experimental procedure are compared to results from self-heating fatigue tests. For specimens orientated at $0^{\circ}$ relative to the rolled direction, microcrack initiation is controlled by the material matrix and the prediction of the fatigue strength with the self-heating method has been observed to be correct. For specimens orientated at $45^{\circ}$ and $90^{\circ}$, the elongated manganese sulfide inclusion clusters are the origin of crack initiation and the fatigue strength drops significantly. For this case, it appears that the self-heating method has difficulty predicting the fatigue behavior.
\end{abstract}

Keywords: anisotropy, forged steel, manganese sulfide inclusions, high cycle fatigue, self-heating

\section{Nomenclature}

$T_{0}:$ ambient temperature

\footnotetext{
*Corresponding author. Tel.: +33 2412073 35; fax: +33241207320.

Email address: etienne.pessard@angers.ensam.fr (Etienne Pessard)
} 
$T:$ average stabilized temperature

$\Sigma_{0}:$ stress amplitude

$\bar{\theta}\left(\Sigma_{0}\right)$ : steady-state temperature variation fot a stress level $\Sigma_{0}$

$k$ : number of active sites

$P_{k}(\Omega)$ : probability of finding $k$ active sites in a domain of volume $V$

$N(\Omega)$ : average number of actived sites

$\lambda$ : intensity of the Poisson Point Process

$m$ : Weibull exponent

$S_{0}$ : scale factor of the Weibull law

$V_{0}$ : specimen volume

$f_{r}$ : cyclic load frequency

$c$ : specific heat capacity

$\tau_{e q}:$ constant depending on the heat transfer boundary conditions

$h$ : hardening modulus

$\rho$ : mass density

$\Delta$ : global dissipated energy density per cycle

$\overline{S_{-1}}$ : mean fatigue limit under purely reversed tensile loads (push-pull)

$\overline{\overline{S_{-1}}}$ : standard deviation of the fatigue limit under purely reversed tensile loads (push-pull)

$P_{F}$ : failure probability

\section{Introduction}

One of the major concerns in designing forged components is the role of nonmetallic inclusions on the fatigue damage mechanisms. Although the influence of non-metallic inclusions on the ductility and toughness of steel is recognized [1, 2, 3, 4, 5, 6, 7, 8, 9, the effect on fatigue properties remains a subject of debate $[8,9,10,11,12,13,14$. The ductility is known to be intimately related to both the volume fraction as well as the morphology of inclusions. Inclusions, such as sulfides and silicates, are usually responsible for drastic reductions in ductility. At the microscopic level, non-metallic inclusions are responsible for the creation of micro-voids, leading to microcracking by coalescence. Hot-rolled and forged steels typically have an anisotropic microstructure and contain manganese sulfide $(\mathrm{MnS})$ inclusions elongated in the laminate direction. The effect of the elongated $\mathrm{MnS}$ inclusions on fatigue strength depends directly on their concentration, morphology and orientation with respect to the applied loads [9. More exactly, several factors, like the shape, size, spatial distribution and adhesion at the inclusion/matrix interface are known to control the fatigue properties. This results in anisotropy of both the fatigue and the monotonic properties. Typically, the high cycle fatigue properties of materials are estimated by conducting cyclic tests on plain specimens. However, due to the high scatter observed around the fatigue limit, many tests are required to obtain a good estimation of the fatigue strength. Hence, this method is often seen as being time consuming and expensive. For example, when using the staircase method [15] it is generally recognized that at least 15 specimens are necessary 
in order to reliably estimate the average value and the standard deviation of the fatigue limit. In the last twenty years, techniques based on self-heating cyclic tests, carried out on a single specimen, have been developed. These methods can provide a rapid estimate of the fatigue strength of metallic materials. Depending on the approach, the average fatigue limit can be estimated [16, 17] and if certain assumptions are made the related standard deviation can also be estimated [18, 19].

This work was undertaken firstly to examine the effect of the inclusion orientation on the tensile strength, impact properties and high cycle fatigue properties of a high strength steel with a bainitic microstructure. The second objective was to investigate the ability of various experimental techniques to reflect the anisotropic behavior of a forged steel under cyclic loading. This work has been performed within the framework of a French ANR (National Research Agency) project titled Optiforge. This paper is the continuation of the work presented in Pessard et al. 14, but has much greater focus on the different experimental methods investigated.

\section{Material and experimental procedure}

The material studied in this work is a Bainitic steel commercially referred to as Metasco MC (25MnCRSiVB6) and is commonly used for the hot forging of automotive components. Its chemical composition is given in table 1 . The material is produced in the form of bars with a reduction ratio of approximately 15. This material is known for its machinability and its good mechanical properties in fatigue. Specimens for monotonic tests, charpy tests and fatigue tests were machined from rolled bars (diameter $80 \mathrm{~mm}$ ) in three different directions, with respect to the rolling direction: parallel $\left(0^{\circ}\right)$, perpendicular $\left(90^{\circ}\right)$ and 45 degrees $\left(45^{\circ}\right)$ (Fig. 1 (b)).

\begin{tabular}{c|cccccccc} 
Element & $\mathrm{C}$ & $\mathrm{Mn}$ & $\mathrm{Si}$ & $\mathrm{Cr}$ & $\mathrm{S}$ & $\mathrm{Mo}$ & $\mathrm{V}$ & $\mathrm{Ti}$ \\
\hline Weight \% & 0.255 & 1.30 & 0.9 & 0.8 & 0.075 & 0.075 & 0.185 & 0.025 \\
Table 1: Chemical composition of the Metasco MC &
\end{tabular}

Micrographic observations suggest that the ex-austenite grains are equiaxed (Fig. 1(a)). This has been confirmed by the Electron Back Scatter Diffraction (EBSD) technique. The EBSD map shows that the bainitic microstructure contains around $10 \%$ austenite (Fig. 2(a)). As each colour of the euler map (2(b)) is associated with a crystallographic orientation, it is possible to estimate the shape and the size of the ex-austenitic grains $(20 \mu \mathrm{m}$ to $50 \mu \mathrm{m})$. Moreover, poles figures (Fig. 3) obtained from EBSD on the residual austenite and ferrite show that there is no crystallographic texture.

These observations indicate that if the mechanical behavior is anisotropic, it cannot be due to the microstructure of the material matrix.

The bainitic microstructure includes inclusions of type MnS, Al2O3, TiN, and VN. The majority of these inclusions are manganese sulfide inclusions that 


\begin{tabular}{ccc} 
& $\begin{array}{c}\text { Individual } \\
\text { MnS Inclusions }\end{array}$ & $\begin{array}{c}\text { MnS } \\
\text { Inclusions Bands }\end{array}$ \\
\hline $\begin{array}{c}\text { Number of } \\
\text { incluions measured }\end{array}$ & 1493 & 300 \\
\hline Diameter $(\mu \mathrm{m})$ & $1.3 \pm 0.6$ & $35 \pm 0.15$
\end{tabular}

Table 2: Diameter of incluions alone and inclusions bands
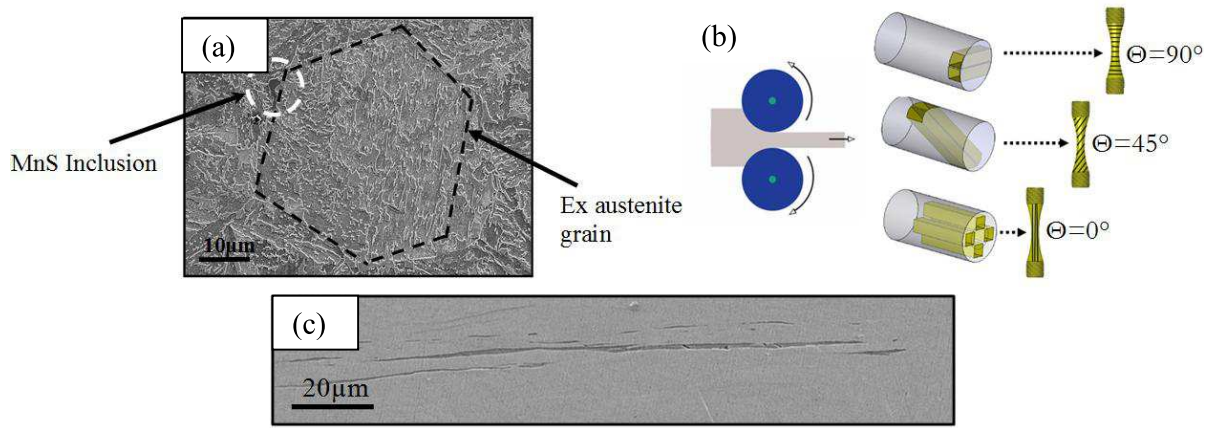

Fig. 1: (a)microstructure of the Metasco MC, (b) extraction of the samples, (c) elongated manganese sulfide inclusion

are elongated in the direction of rolling (Fig. 11(c)). An X-Ray Tomography analysis was conducted to observe the inclusion geometry in 3D. As shown in Fig. 4. the inclusions are grouped into bands or clusters, which can be very long. The diameter of individual inclusions and inclusion bands measured from SEM images are presented in Table 2. The average shape ratio (length/width) of individual inclusions is approximately seven, and is greater than 30 for the largest inclusions. The volume fraction of manganese sulfide inclusions, determined via tomography, is approximately $0.5 \%$. Note that the biggest inclusions ( $1 / 3$ by number) represent $85 \%$ of the total inclusion volume.

Microscopics observations shows that the fibering is composed of inclusions bands. The objective of the next section is to study the effect of the elongated inclusions bands on different mechanical properties of the material.

\section{Experimental results}

\subsection{Monotonic mechanical properties of the bainitic forged steel}

Monotonic tensile tests were carried out for each fibering orientation. Typical results are shown in Table 3. The Ultimate Tensile Strengths (UTS) of the three orientations are very similar. The UTS is slightly higher for a fibering oriented at $0^{\circ}$ and equal for orientation at $45^{\circ}$ and $90^{\circ}$. The tensile elongation shows greater dependence on the fibering angle and decreases with increasing fibering orientation angle. 


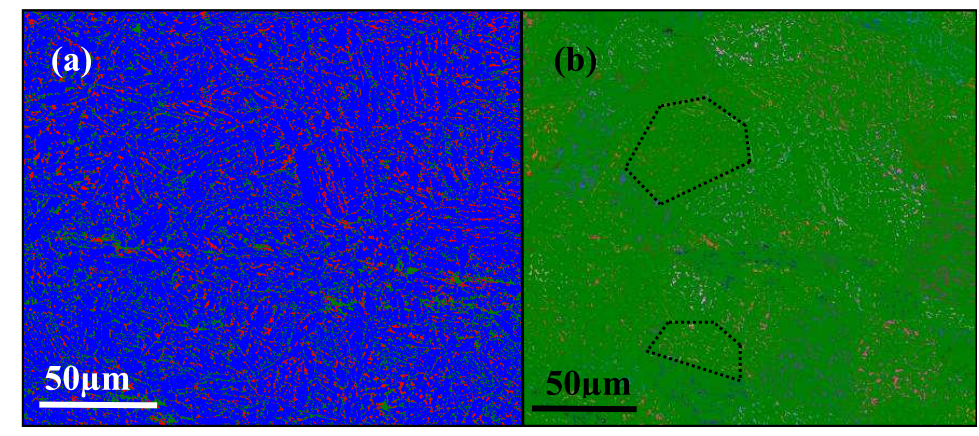

Fig. 2: (a)Phase map (red=austenite, blue=ferrite), (b)Euler map of retained austenite
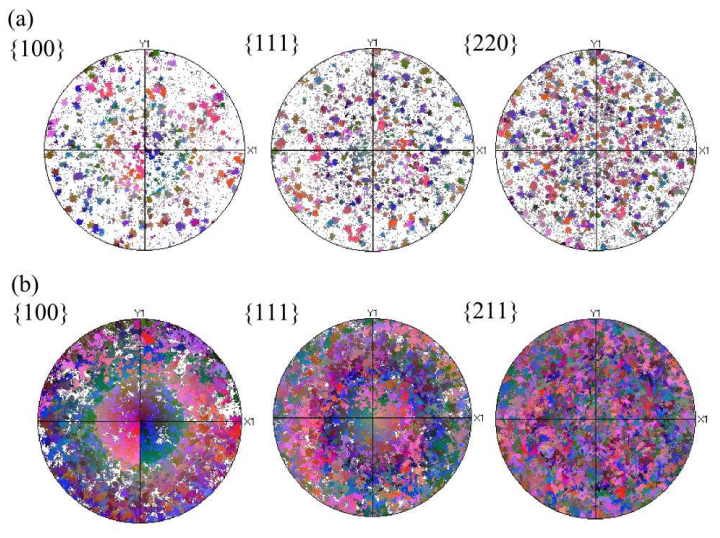

Fig. 3: Poles figures (a) of austenite, (b) of ferrite

For specimens with a fibering orientation of $0^{\circ}$, examination of the fracture surfaces of failed tensile specimens showed that the fracture mode was ductile: microscopic ductile dimples localized near the specimen surface can be observed (Fig. 5(a) and (b)). For specimens with a fibering orientation of $45^{\circ}$ and $90^{\circ}$, ductile dimples are observed only around inclusions. Far from the inclusion the fracture surface indicates a brittle failure mode (Fig. 5(c) and (f)). This analysis of the failure surface is in accordance with the quantitative results presented in Table 3 .

\subsection{Toughness of the bainitic forged steel}

The toughness of the steel has been measured by the Charpy V-notch (CVN) impact energy in accordance with the standard NF EN 10045 1. Fig. ?? shows the impact energy plotted as a function of temperature to observe the ductile to brittle transition. This type of test is sometimes used to detect the effect of inclusions on the mechanical behavior of steels 9 . For the specimens with a 


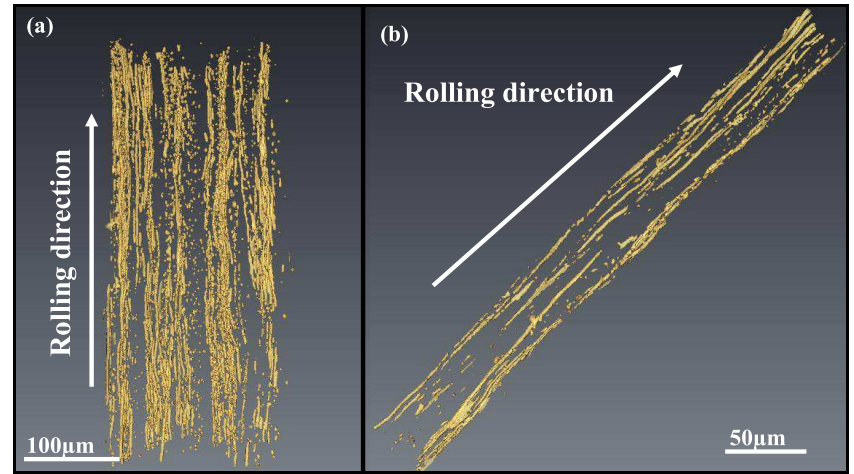

Fig. 4: 3D geometry of MnS inclusion after tomography observation (a) several inclusions, (b) inclusion band isolated

\begin{tabular}{cccc}
$\begin{array}{c}\text { Orientation } \\
\text { (degrees) }\end{array}$ & $\begin{array}{c}\text { Yield Stress } \\
\text { YS(MPa) }\end{array}$ & $\begin{array}{c}\text { Ultimate Tensile Stress } \\
\text { UTS (MPa) }\end{array}$ & $\begin{array}{c}\text { Tensile Elongation } \\
\text { TE\% }\end{array}$ \\
\hline $0^{\circ}$ & 755 & 1180 & 25 \\
$45^{\circ}$ & 710 & 1127 & 14 \\
$90^{\circ}$ & 750 & 1130 & 5
\end{tabular}

Table 3: Diameter of incluions alone and inclusions bands

fibering orientation of $0^{\circ}$ the fracture toughness increases with the temperature: a ductile to brittle transition is observed. For $45^{\circ}$ and $90^{\circ}$ specimens the fracture toughness is effectively independent of the temperature.

Examination of the fracture surfaces of broken Charpy impact specimens at $-40^{\circ} \mathrm{C}$ showed that the fracture mode was brittle. Crack initiation for fibering orientations of $45^{\circ}$ and $90^{\circ}$ is localized at inclusions bands (Fig. 77(b) and (c)), whereas at $0^{\circ}$ cracks appear to initiate from the bainitic matrix (Fig. 7 (a)). At $20^{\circ} \mathrm{C}$ the results are very different: the fracture surface is ductile for fibering orientations of $0^{\circ}$ but brittle for specimens at $45^{\circ}$ and $90^{\circ}$. At $20^{\circ} \mathrm{C}$, the evolution of the mechanisms with respect to the fibering orientation explains the anisotropic fracture toughness observed in Fig. 7.

\subsection{Anisotropic fatigue behavior}

\subsubsection{Conventional fatigue tests}

Push Pull ( $\mathrm{R}=-1)$ fatigue tests were performed using a servo hydraulic Instron testing machine at a frequency of $25 \mathrm{~Hz}$. The geometry of the non-standard fatigue specimens are presented in Fig. 8. The fatigue limits at $2 \times 10^{6}$ cycles were evaluated using the staircase method. All tests were carried out at room temperature and 15 specimens for each orientation have been used to define the average fatigue limit and the scatter. 

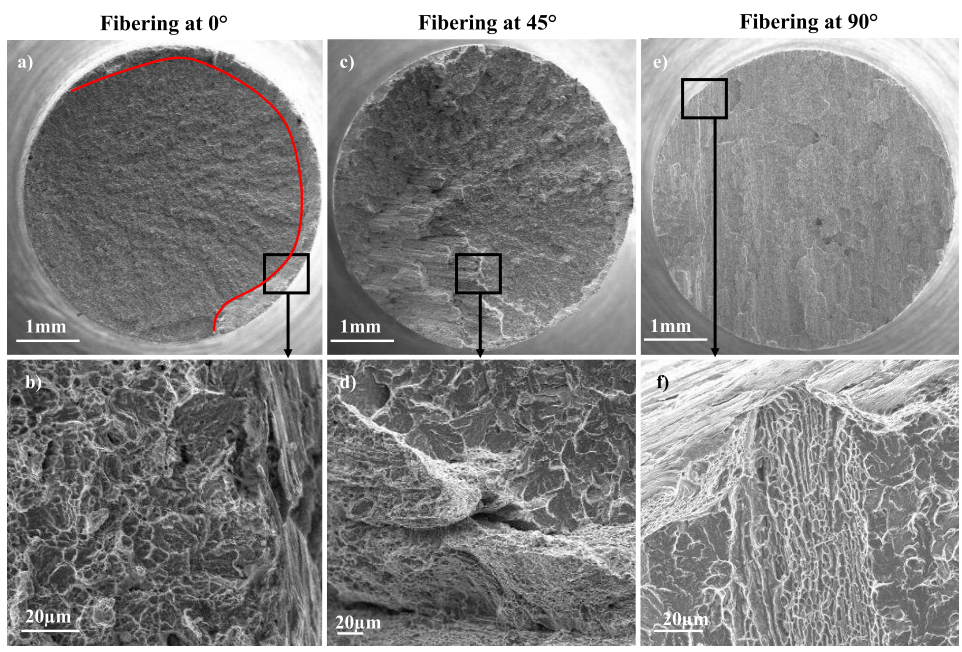

Fig. 5: Fracture surface of specimens broken during monotonic tensile test: (a) and (b) fibering at $0^{\circ},(\mathrm{c})$ and (d) fibering at $45^{\circ},(\mathrm{e})$ and (f) fibering at $90^{\circ}$

The results obtained are presented in Fig. 9 and Table 4 . As observed in the literature 9, 12 for steels whose fatigue behavior is controlled by defects, the fatigue limit (or fatigue strength at $2 \times 10^{6}$ cycles) is highest for an orientation of $0^{\circ}$ and lowest at $90^{\circ}$. Note that the decrease of the fatigue strength is accompanied by a reduction of the standard deviation. This result follows the same trend as those obtained by Temmel 12 for a 50CrMo4 steel.

\begin{tabular}{cccc}
$\begin{array}{c}\text { Orientation } \\
\text { (degrees) }\end{array}$ & $\begin{array}{c}\text { Average Fatigue Strength } \\
\overline{S_{-1}}\end{array}$ & $\begin{array}{c}\text { Standard Deviation } \\
\overline{S_{-1}}\end{array}$ & $\begin{array}{c}\text { Covariance } \\
\text { Cov }=\overline{\overline{S_{-1}}} / \overline{S_{-1}}\end{array}$ \\
\hline $0^{\circ}$ & 476 & \pm 20 & 0.042 \\
$45^{\circ}$ & 453 & \pm 8 & 0.0018 \\
$90^{\circ}$ & 415 & \pm 11 & 0.026
\end{tabular}

Table 4: Push pull fatigue test results

Examination of the fracture surfaces of broken fatigue specimens showed that the origin of fracture depends on the fibering orientation. Crack initiation for a fibering orientation of $45^{\circ}$ and $90^{\circ}$ is localized at inclusions bands (Fig. $10(d)$ and (f)), and for an orientation of $0^{\circ}$ it seems to be initiated from the bainitic matrix ((Fig. 10(b)). For this case, the origin of fracture is similar to those shown by the Charpy tests at $20^{\circ} \mathrm{C}$.

\subsubsection{Anisotropic fatigue crack mechanisms at the mesoscopic scale}

In-situ observations were conducted using specimens (3 for each orientation) on which a flat area was machined and polished (using $1 \mu \mathrm{m}$ diamond paste). 

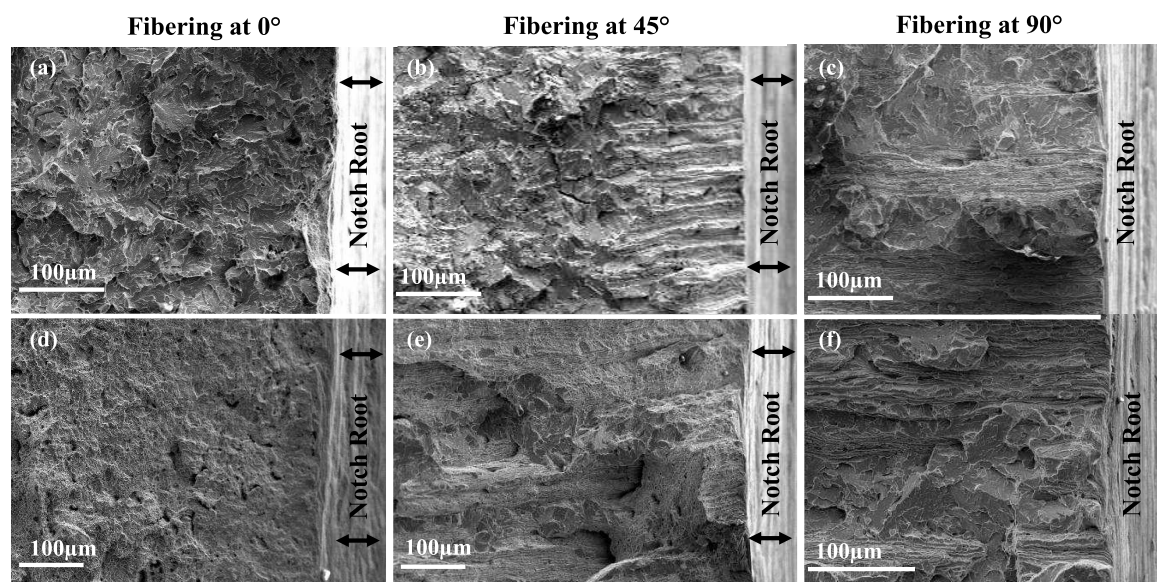

Fig. 6: Fracture surface of specimens broken during impact tests: (a), (b) and (c) Charpy tests at $-40^{\circ} \mathrm{C},(\mathrm{d}),(\mathrm{e})$ and (f) Charpy tests at $20^{\circ} \mathrm{C}$

These surface observations were done, using a long range optical microscope, during fatigue tests in which the specimens were loaded at the previously determined average fatigue limit. The aim was to determine the nucleation and orientation of surface micro-cracks.

For the $\mathbf{0}^{\circ}$ specimens, where the inclusions are parallel to the applied stress, the cracks initiate in the material matrix (i.e. the bainitic microstructure), typically at $45^{\circ}$ to the loading direction (Fig 11(a)). This corresponds to the classical fatigue crack initiation mechanism in which micro-cracks form on a critical plane (or plane of greatest shear stress amplitude), in the weakest and/or most favorably orientated grains. Both in-situ observation of the specimen surfaces (Fig. 11) and SEM observations of the failure surfaces (Fig. 10(a) and (b)) show that the crack initiation sites are not associated with the presence of non-metallic inclusions. The bainitic microstructure governs microcrack growth: it can be observed in (Fig. 11(a)) that a crack is blocked by a bainite latte and in (Fig. 11(b)) another crack branches due to the presence of a bainite latte.

For the $\mathbf{9 0}^{\circ}$ specimens, observations show that there are two different fatigue crack initiation mechanisms that can occur. These are:

- Crack initiation in the material matrix as discussed above for the $0^{\circ}$ case, (see the bottom right of Fig. 12).

- Crack initiation from Manganese Sulphide (MnS) inclusion clusters (see the top left of Fig. 12). The inclusion type was verified by EDS (Energy Dispersive Spectrometry). It can be seen that these soft, non-metallic inclusions are elongated in the direction of rolling. However, more importantly, these inclusions are grouped into bands or clusters (See Fig. 4), inducing a detrimental effect on the fatigue strength. The cracks initiate 

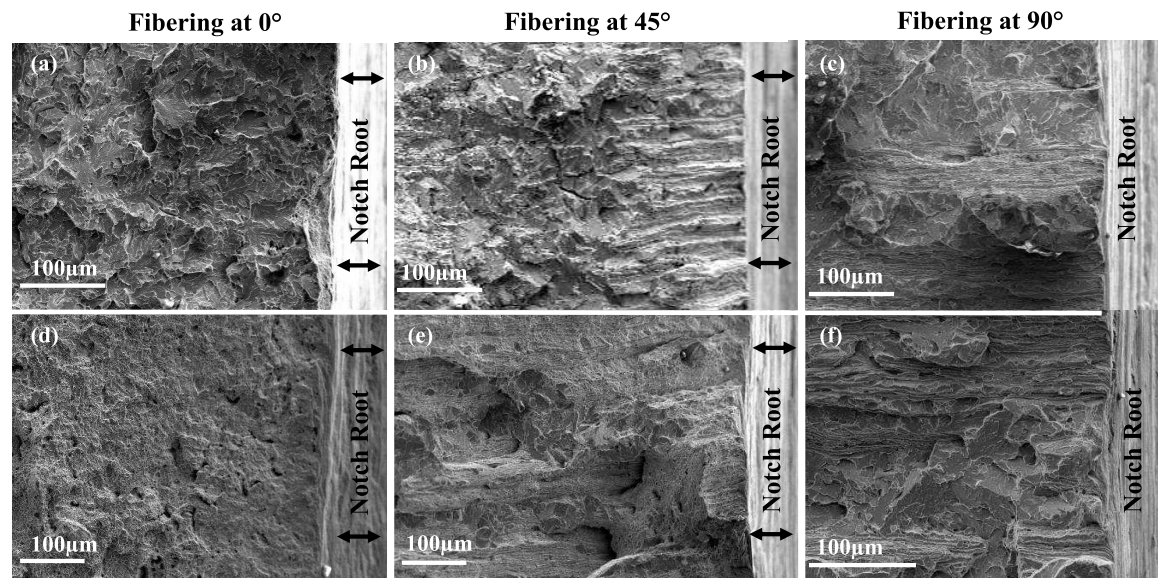

Fig. 7: Fracture surface of specimens broken during impact tests: (a), (b) and (c) Charpy tests at $-40^{\circ} \mathrm{C},(\mathrm{d}),(\mathrm{e})$ and (f) Charpy tests at $20^{\circ} \mathrm{C}$

at the edges of the biggest inclusions and then propagate in the direction of the neighboring inclusions (Fig. 13) in the band. The crack can propagate along the inclusion interface or through inclusions (Fig. 13.

For the $90^{\circ}$ orientation, final failure of the specimens is determined via competition between these two mechanisms.

All these observations show that the orientation of the specimen, with respect to the rolling direction, greatly influences the fatigue crack mechanisms. At $0^{\circ}$ the cracks are initiated in the material matrix and both the fatigue limit and the standard deviation of the fatigue limit are high. At $90^{\circ}$, the $\mathrm{MnS}$ clusters appear on the fracture surface as bands, which govern the fatigue behavior and further decrease the fatigue limit and the standard deviation.

For the $45^{\circ}$ specimens, the fatigue crack initiation mechanisms are very similar to the $90^{\circ}$ case.

\subsection{Rapid estimation of fatigue strength by self-heating measurements}

Over the last twenty years certain authors [16, 17, 18, 19, 27, 28, have proposed an alternative way to estimate the high cycle fatigue strength, of metallic materials, by using temperature measurements. This method involves the observation of the thermal activity during cyclic loading. The main advantages are that only one specimen is required and the testing time is considerably shorter when compared to traditional high cycle fatigue tests like the staircase method. The method typically requires the cyclic loading of the test piece or specimen in a series of constant amplitude block loads, in which the stress amplitude, $\Sigma_{0}$, for each block is successively increased. For each stress amplitude (or each block), the change of the temperature, $\theta=T-T_{0}$ is recorded, where $T$ is the average stabilized temperature on the surface of the specimen and $T_{0}$ is the 


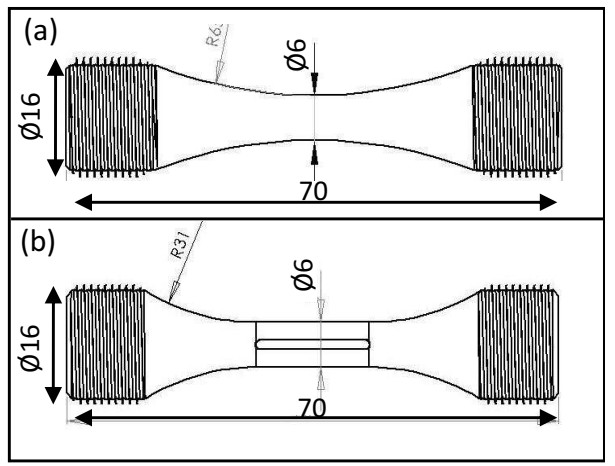

Fig. 8: Geometry of fatigue specimens used: (a) for stair case and (b) for observation and self heating tests (units in $\mathrm{mm}$ )

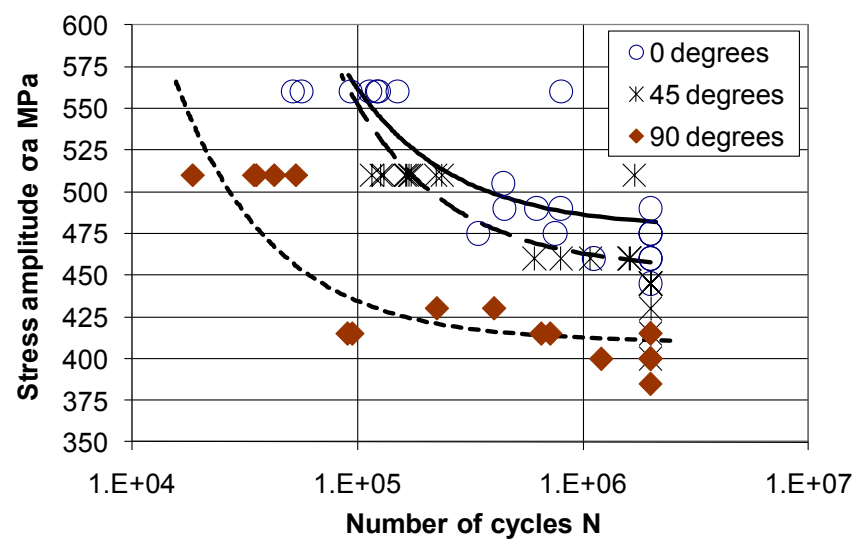

Fig. 9: SN curve obtained for each fibering orientation

ambient temperature. For each block, the average temperature stabilizes (or reaches steady state) after a certain number of cycles depending on the stress level and the loading frequency and is noted as $\bar{\theta}\left(\Sigma_{0}\right)$. It is observed that beyond a given stress level, close to the fatigue limit, the steady state temperature, $\bar{\theta}\left(\Sigma_{0}\right)$, starts to increase significantly. This regime change, corresponding to the activation of micro-plasticity mechanisms, gives a rapid empirical identification method to estimate the mean fatigue limit [16, 17]. As a consequence, some authors have proposed that the fatigue limit can be determined by plotting the stabilization temperature against the applied stress and determining the value of the fatigue limit by the change in slope of this curve. This empirical method has been tested for smooth and notched specimens and provides good results for the estimation of the average fatigue limit with an error of less than $10 \%$ [17. 


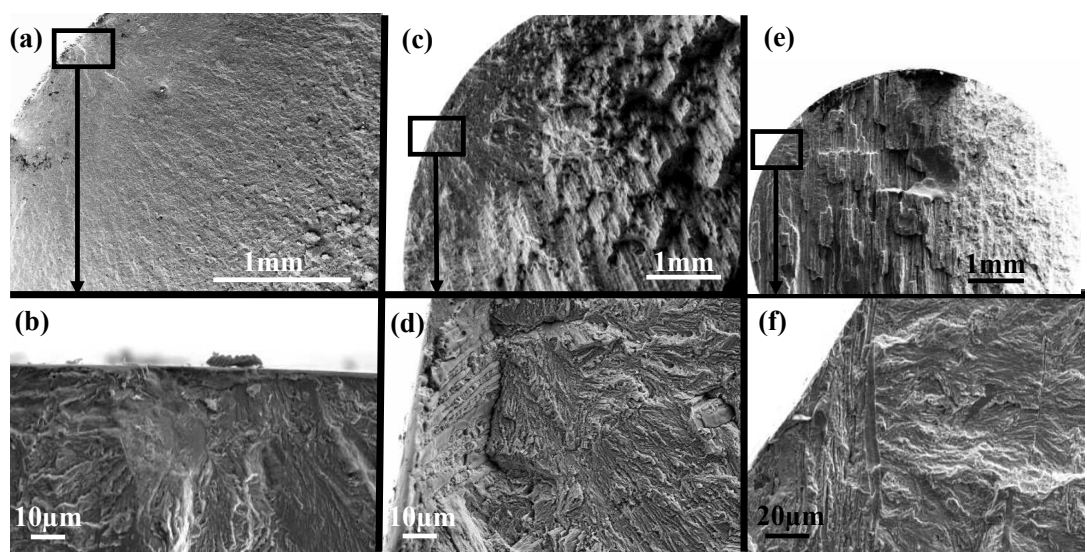

Fig. 10: Fracture surface of specimens broken during push pull fatigue test: (a) and (b) fibering at $0^{\circ},(\mathrm{c})$ and (d) fibering at $45^{\circ}$, (e) and (f) fibering at $90^{\circ}$

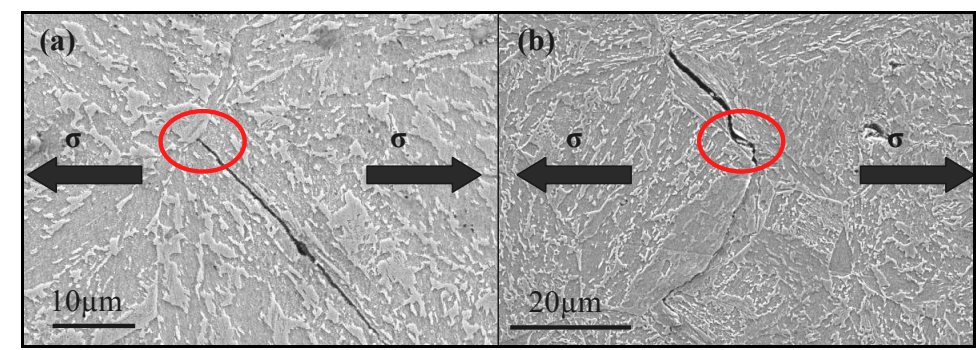

Fig. 11: Fibering oriented at $0^{\circ}$, microcrack in the bainite, effect of microstructure on the growth on the specimen surface

More recently Doudard et al 18, 19, proposed a probabilistic model that is able to define the scatter in high cycle fatigue from the self-heating curve. More precisely, an original identification procedure is employed to determine the scatter of experimental fatigue results by assuming that it is induced by heterogeneous micro-plastic activity. This method uses the Eshelby localization rule and describes, at the local scale, the appearance of micro-plasticity by considering a set of elasto-plastic grains, referred to as inclusions, randomly distributed within an elastic matrix. The principal hypothesis of this model considers that micro-plasticity is the origin of fatigue damage and that it is the principal source responsible for the observed self-heating.

The objective of the work presented in this section is to verify if it is possible, by using self-heating measurements, to estimate the average fatigue limit and the related scatter of the bainitic steel Metasco MC under investigation and in particular for each fibering orientation. This high strength steel has been shown in the previous section, to behave in an anisotropic manner under cyclic 


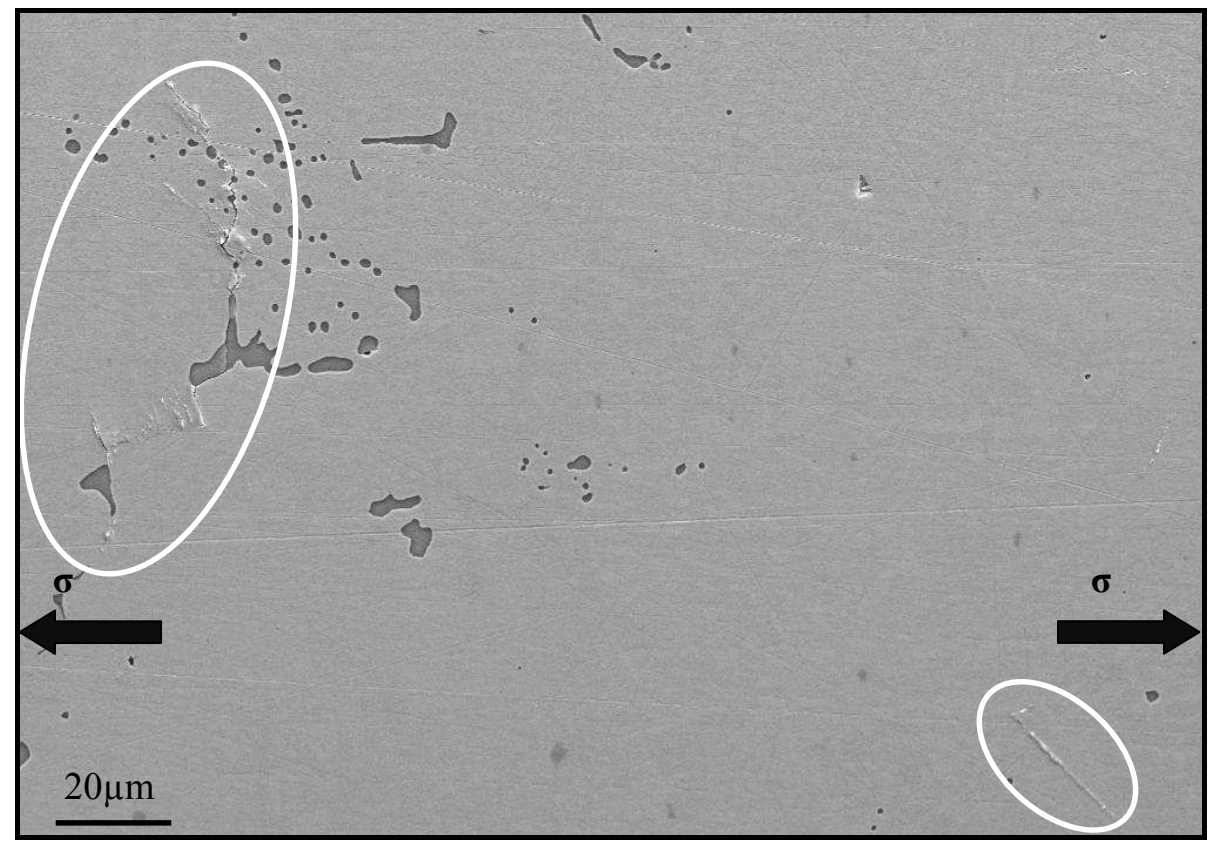

Fig. 12: Observation on the surface at $90^{\circ}$, competition between the two mechanisms

loading. Therefore, the question is: are the self-heating methods able to reflect this anisotropic behavior?

\subsubsection{Self-heating tests}

To check the repeatability of the method, 3 specimens for each fibering orientation have been tested in fatigue. In addition, tests were conducted in two different laboratories. Two of the 3 specimens (for each orientation) were tested under the same conditions in the LAMPA ${ }^{a}$ laboratory and one set of specimens was tested at the $\mathrm{CREAS}^{c}$ laboratory using the same cyclic loading block sequences, but with different equipment. Note that the two laboratories used servo-hydraulic fatigue testing machines with different grips and temperature acquisition systems. The load ratio used in the tests is $R=-1$. A single type $T$ thermocouple is fixed to the specimen surface via adhesive tape. The accuracy of the temperature measurements is greater than $0.02^{\circ} \mathrm{C}$. In order to compare the results from the self-heating tests and from the staircase method the specimen geometries used are very similar (Fig. 8(a) and (b)).

The evolution of the temperature on the specimen surface for different stress amplitudes is shown in Fig. 14. For low stress amplitudes $(\sigma \leq 500 \mathrm{MPa})$ the temperature stabilizes quickly, after 1000 cycles (Fig. 14(a) and (b)). For higher applied stress amplitude $(\sigma \geq 500 \mathrm{MPa})$ the stabilization is not clearly observed and after 1000 cycles the temperature continues to slowly increase (Fig. 14(c)). 


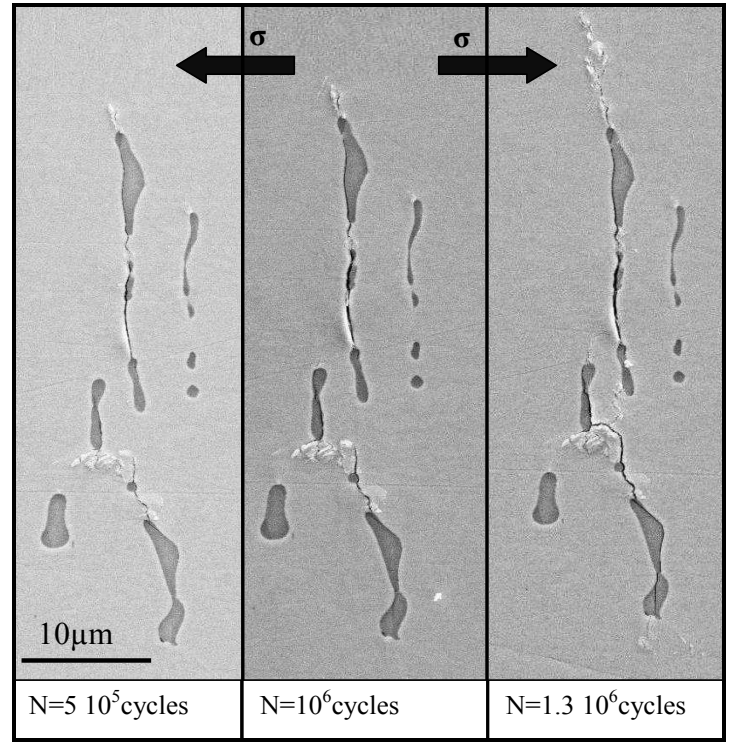

Fig. 13: Crack path on inclusions band for a fibering oriented at $90^{\circ}$

Fig. 15 shows the self-heating curves for different fibering orientations. The curves are very similar and seems to be independ of the fibering orientation. Fig. 15(a) and (b) shows a comparison of the self-heating curves measured by the CREAS and the LAMPA laboratories. The curves obtained are similar and have the same trend, note that all the curves obtained by CREAS show slightly lower temperatures than the LAMPA curves. The curves of the two batches of tests realized by LAMPA are identical.

\subsubsection{Prediction of fatigue properties}

To estimate the average fatigue limit, the iteration procedure developed by Cura [17] is used. This method is based on the graphical representation described by Luong [16. The fatigue limit is defining as the intersection of the two straight lines interpolating the experimental data (see Fig. 16(a)). This iteration method allows a rapid evaluation of the intersection point, when the fatigue limit is, a priori, unknown.

To estimate the scatter, the probabilistic model of Doudard [18, 19] is used. The microscopic yield stress is considered as a probabilistic variable. The authors define the notion of an active site where micro-plasticity and heat generation occurs. The apparition of these sites is supposed to follow a specific Poisson Point Process (PPP). The probability of finding $\mathrm{k}$ active sites in a domain of volume $\mathrm{V}$ is given by:

$$
P_{k}(\Omega)=\frac{[-N(\Omega)]^{k}}{k !} \exp [-N(\Omega)]
$$



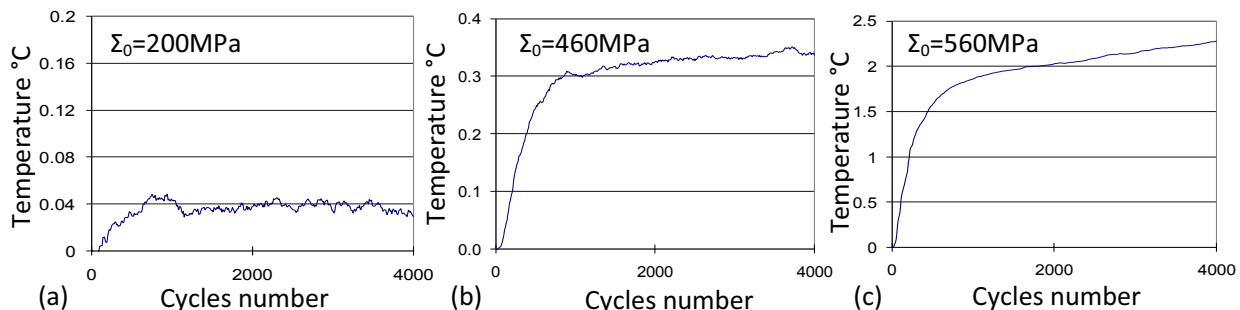

Fig. 14: Temperature evolution during 4000 cycles for different stress amplitudes using the Metasco MC , fibering orientated at $45^{\circ}, \mathrm{f}=10 \mathrm{~Hz}, \mathrm{R}=-1$, (a) $\Sigma_{0}=200 \mathrm{MPa}$, (b) $\Sigma_{0}=460 \mathrm{MPa}$ and (c) $\Sigma_{0}=560 \mathrm{MPa}$
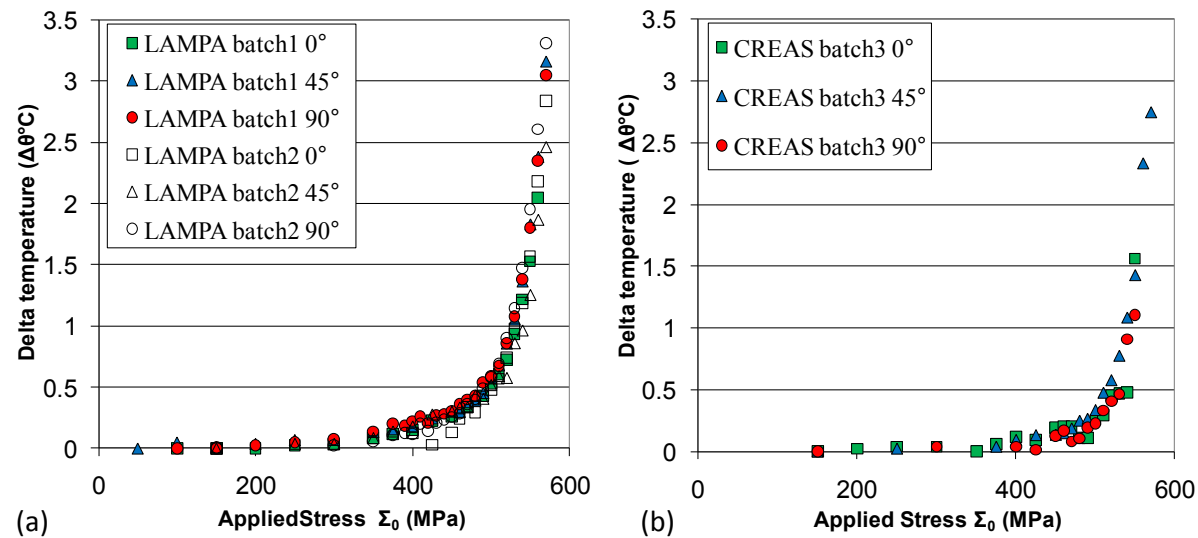

Fig. 15: Comparison of the self heating curves for different fibering orientations, $R=-1, f=10$ $\mathrm{Hz}$, (a) measured by the LAMPA laboratory and (b) measured by the CREAS laboratory

Where $N(\Omega)$ is the average number of activated sites in the domain $\Omega$ defined by:

$$
N(\Omega)=\lambda V_{\Omega}
$$

With $\lambda$ the intensity of the process also equal to the density of active sites. The authors [18, 19] chose a power law of the applied stress amplitude to define the process intensity:

$$
\lambda\left(\Sigma_{0}\right)=\frac{1}{V_{0}}\left(\frac{\Sigma_{0}}{S_{0}}\right)^{m}
$$

Where $S_{0}$ is a scale factor and $m$ is the Weibull exponent reflecting the scatter in micro-plasticity activity.

The plastic strain energy at a site and in the domain $\Omega$ of volume $V_{\Omega}$ are estimated by using the concepts of continuum thermodynamics and by estimating the number of activated sites by means of the Poisson Point Process. A Kröner 

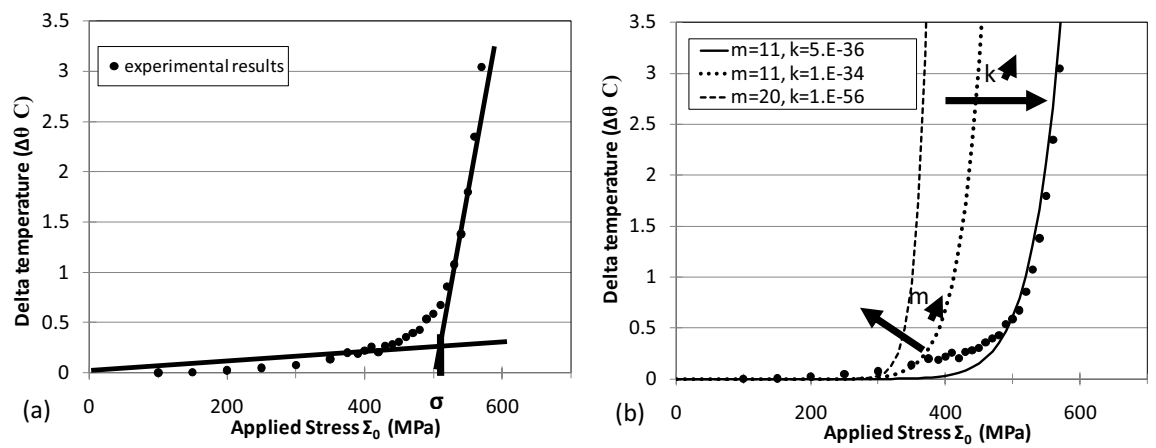

Fig. 16: (a) Estimation of the fatigue limit using the Cura Method [17 and (b) estimation of the $m$ exponent using Doudard method 19]

type localization rule 20. together with a plastic material model including combined kinematic and isotropic hardening, allow the evaluation of the plastic energy at a site. The temperature evolution can be determined by analyzing the heat transfer during the cyclic loading. The heat conduction equation [18] can be written as:

$$
\dot{\theta}+\frac{\theta}{\tau_{e q}}=\frac{\Delta f_{r}}{\rho c}
$$

Where $\tau_{e q}$ is a constant depending on the heat transfer boundary conditions, $c$ is the specific heat capacity and $f_{r}$ the cyclic load frequency. Equation (4) shows that the global dissipated energy density per cycle $\Delta$, influences directly the average temperature evolution. The resolution of the heat equation (4) leads to the evolution of the stabilized temperature, as a function of the applied stress amplitude, $\Sigma_{0}$ [21]. When the temperature stabilizes, (i.e. the dissipation per cycle $\Delta$ is constant), the change of the steady state temperature with the stress amplitude is calculated by solving equation (4):

$$
\bar{\theta}=\frac{\Delta f_{r} \tau_{e q}}{\rho c}=\eta V_{0} \frac{m}{(m+1)(m+2)} \frac{\Sigma_{0}^{m+2}}{\left(S_{0} V_{0}^{1 / m}\right)^{m}}
$$

Hence, the model depends on three quantities, $S_{0} V_{0}^{1 / m}$ (a scale parameter of the Weibull model), $m$ (the Weibull exponent) and $\eta V_{0}$ (the scale parameter of the temperature variation) with:

$$
\eta=\frac{4 f_{r} \tau_{e q}}{h \rho c}
$$

In this experimental investigation, the material, the frequency and the thermal boundary conditions are the same and therefore the three quantities can be considered to be constant. Hence equation (5) can be simplified as:

$$
\bar{\theta}=K \Sigma_{0}^{m+2}
$$


Within the weakest link theory [23], the failure probability $P_{F}$ of a domain $\Omega$ of volume $V_{\Omega}$ under a homogeneous stress amplitude $\Sigma_{0}$ is equal to the probability of finding at least one active inclusion in the volume $V_{\Omega}$. Using these hypotheses the failure probability obtained is finally a Weibull law:

$$
P_{F}=1-\exp \left[\frac{V_{\Omega}}{V_{0}}\left(\frac{\Sigma_{0}}{S_{0}}\right)^{m}\right]
$$

Where $V_{0} S_{0}^{m}$ is a material parameter. To obtain the Weibull exponent of the Doudard model, the self-heating curve is approximated by expression (7). The experimental Weibull exponent can also be directly determined by using the staircase results [19, using the mean fatigue limit $\overline{S_{-1}}$ and the corresponding variance $\overline{\overline{S_{-1}}}$ :

$$
C V=\frac{\overline{\overline{S_{-1}}}}{\overline{S_{-1}}}=\frac{\sqrt{\Gamma\left[1+\frac{2}{m}\right]-\Gamma^{2}\left[1+\frac{1}{m}\right]}}{\Gamma\left[1+\frac{1}{m}\right]}
$$

Where $\Gamma(t)=\int_{0}^{\infty} x^{t-1} e^{-x}$ is the gamma function [23] and CV is the covariance.

Table 5 shows the comparison of the mean fatigue limit and the Weibull exponent estimated using the staircase method and the self-heating method. The predictions from tests performed in the LAMPA and the CREAS laboratories are very close, which validates the experimental protocol. In the following it is assumed that the correct result is obtained by the staircase method. For a fibering orientation of $0^{\circ}$, the mean fatigue limit is correctly estimated by the self-heating method with an error of $5 \%$. The Weibull exponent estimated from the self-heating test is consistently lower $(>100 \%)$ than the value obtained from the staircase method. The most important conclusion that can be made from Table 5 is that the self-heating method does not appear to be able to detect the anisotropic fatigue behavior of the Metasco MC steel. In fact, the mean fatigue limit and the corresponding Weibull exponent remain constant for all fibering orientations.

\begin{tabular}{c|cc|cc|cc} 
& $\begin{array}{c}\text { LAMPA Results } \\
\text { (2 specimens for } \\
\text { each orientation })\end{array}$ & \multicolumn{2}{|c|}{$\begin{array}{c}\text { CREAS Results } \\
\text { (1 specimen for } \\
\text { each orientation })\end{array}$} & $\begin{array}{c}\text { Staircase Results } \\
\text { (15 specimens for } \\
\text { each orientation })\end{array}$ \\
\hline Orientation & $\overline{S_{-1}}(\mathrm{MPa})$ & $\mathrm{m}$ & $\overline{S_{-1}}(\mathrm{MPa})$ & $\mathrm{m}$ & $\overline{S_{-1}}(\mathrm{MPa})$ & $\mathrm{m}$ \\
\hline $0^{\circ}$ & 491 & 10 & 502 & 11 & 476 & 30 \\
$45^{\circ}$ & 493 & 11 & 503 & 14 & 453 & $>50$ \\
$0^{\circ}$ & 501 & 11 & 490 & 14 & 415 & 45
\end{tabular}

Table 5: Comparison between results from self heating and staircase methods 


\section{Discussions}

The experimental tests discussed in this article can all be used for the mechanical characterization of steel materials. However, the focus of this work is to investigate the ability of these tests to predict the anisotropic nature of a particular forged steel (which is primarily due to elongated MnS inclusions). Concerning the monotonic tensile tests, the yield stress and the ultimate tensile strength are not really affected by the inclusion orientation, whereas the tensile elongation percent is strongly affected. The fracture toughness (or Charpy energy) is the material parameter that is the most sensitive to the inclusion orientation, which is in agreement with the results published by Wilson 3. The inclusion orientation can facilitate crack propagation, decrease the ductility, the fracture toughness and the mean fatigue limit. These conclusions lead to greater understanding of the effect of elongated inclusions on the mechanical behavior of steels and maybe useful for other applications where it is possible to observe the same type of mechanisms (low carbon steels [24, railway wheels 25], high stregth steels for gears 50CrMo4 [13] and 30CrNiMo8 [26]).

Concerning the self-heating tests, the empirical technique proposed by Luong and Cura [16, 17] to estimate the average fatigue limit seems to provide satisfactory results only when the non-metallic inclusions do not participate in the local fatigue damage mechanisms. It has been shown in Section 3.3.2. that the crack initiation mechanism does not remain the same when the fibering orientation with respect to the loading direction changes. For the $90^{\circ}$ orientation, a competition between crack initiation from inclusion clusters and initiation from the bainitic matrix occurs. While for the $0^{\circ}$ orientation, crack initiation is only controlled by the matrix. The results from the staircase method highlight that this change in crack initiation mechanism is associated with a decrease in the average fatigue limit and standard deviation. The role of the inclusion clusters become more pronounced when they are properly oriented to the loading axis and this leads to a detrimental effect on the fatigue strength. This detrimental influence is not captured by the self-heating measurements since the curves for the three fibering orientations are effectively superimposed (see Fig. 15). Therefore, the method estimates very similar average fatigue limits for each orientation. One possible reason for this incapacity to reflect the anisotropic behavior is the simultaneous occurrence of crack initiation sites of different natures. Indeed, even for the $90^{\circ}$ fibering orientation and despite the lowest applied stress level close to the fatigue limit, micro-plasticity in the bainitic microstructure is still active. This activity leads to plastic dissipation resulting in a temperature increase and self-heating. The other initiation sites, (i.e. initiation from inclusion clusters), constitute other sources of plastic dissipation but do not seem to modify the overall dissipation captured through the temperature measurement. However, these inclusions seem to be at the origin of the fatal crack leading to failure. Fig. 12 and 13 clearly show that the cracks, after their initiations at inclusion clusters, grow perpendicular to the specimen axis (in mode I) inside the cluster before attaining a size sufficiently large to cross the microstructural barriers of the bainitic microstructure (lattes of bainite and austenite grain boundaries). 
The capacity of the initiated crack to cross these barriers is then made easier by the presence of the inclusions. Note that Baudry et al. [27] obtained the same type of results for a $100 \mathrm{Cr} 6$ martensitic steel in Torsion. In this study, the estimation of the fatigue limit with the self-heating method is $20 \%$ higher then the estimation with the staircase method. For these authors the self-heating method is only suitable for estimating the fatigue strength of the material matrix and for a 100Cr6 with crack initiation from inclusion the method overestimates the fatigue limit. If the activation scenario proposed by Doudard [18, 19] is employed to model this material, it is incorrect to use only one Poisson Point Process to reflect the probability of finding active sites in a volume. According to our observations, two processes should be used. One for the bainitic matrix and the other one for the inclusion clusters. Of course, the corresponding intensities should not be described by the same power law. In the following, two process intensities are proposed and given by:

$$
P_{k_{1}}(\Omega)=\frac{\left[-N_{1}(\Omega)\right]^{k_{1}}}{k_{1} !} \exp \left[-N_{1}(\Omega)\right] ; P_{k_{2}}(\Omega)=\frac{\left[-N_{2}(\Omega)\right]^{k_{2}}}{k_{2} !} \exp \left[-N_{2}(\Omega)\right]
$$

$k_{1}$ and $k_{2}$ are respectively the number of active sites in the bainitic matrix and around inclusion clusters. Following Doudards work, the global dissipated energy per cycle is now the sum of the contributions from the matrix and the inclusions.

$$
\Delta=\Delta_{1}+\Delta_{2}
$$

In the particular case of constant isotropic hardening, Doudard [18, 19] proposed an expression for the global dissipated energy density per cycle $\Delta$, for a site volume $V_{S}$ :

$$
\Delta=\frac{4 V_{S}}{h} \frac{m}{(m+1)(m+2)} \frac{\Sigma_{0}^{m+2}}{\left(S_{0} V_{0}^{1 / m}\right)^{m}}
$$

Where $h$ is the hardening modulus which is considered constant. Using the heat equation (4) and the global dissipated energy density per cycle (12) for each mechanism, a new expression for the stabilized temperature can be proposed:

$\bar{\theta}=\eta V_{0} \frac{m_{1}}{\left(m_{1}+1\right)\left(m_{1}+2\right)} \frac{\Sigma_{01}^{m_{1}+2}}{\left(S_{01} V_{0}^{1 / m_{1}}\right)^{m_{1}}}+\eta V_{0} \frac{m_{2}}{\left(m_{2}+1\right)\left(m_{2}+2\right)} \frac{\Sigma_{02}^{m_{2}+2}}{\left(S_{02} V_{0}^{1 / m_{2}}\right)^{m_{2}}}$

Where $\Sigma_{01}, \Sigma_{02},\left(m_{1}, S_{01} V_{0}^{1 / m_{1}}\right)$ and $\left(m_{2}, S_{02} V_{0}^{1 / m_{2}}\right)$ are respectively the two equivalent stresses, the Weibull exponents and the scale parameters related to the two observed mechanisms.

The estimation of the dissipated plastic energy due to the inclusion clusters cannot be determined by the first model proposed by Doudard. Indeed, Doudard assumed that the Representative Elementary Volume (REV) contains a set of 
elasto-plastic inclusions, or sites, randomly distributed within an elastic matrix. A Hershey Kröner type localization rule makes it possible to estimate the stress and strain fields in this elasto-plastic inclusion. For the case of an inclusion clusters, this approach seems inappropriate to describe the stress and strain fields around the non-metallic inclusions and to compute the related dissipated energy. Another approach is hence necessary. A recent model proposed as an extension of Doudard work, for cast copper aluminum alloy [28] appears interesting along with experimental techniques developed for measuring the temperature at the local scale with infrared thermography [29].

Nevertheless, since the self-heating measurements do not detect crack initiation from inclusion clusters, it is natural to conclude that the corresponding dissipated energy $\Delta_{2}$ is much smaller than the energy due to the matrix $\Delta_{1}$. In order to apply the proposed model (equation 13) it is necessary to be able to measure the thermal dissipation due to the inclusions. This work is in progress and is based on the use of infra red thermal measurements to quantify the local dissipation at the mesoscopic scale.

Finally by using the weakest link theory and considering that the two types of initiation event are totally independent, the total failure probability is equal to the probability of finding at least one active site either in the matrix or in the inclusion clusters.

$$
P_{F}=P(N>1)=1-\exp \left[-\frac{V_{\Omega}}{V_{0}}\left(\left(\frac{\Sigma_{01}}{S_{01}}\right)^{m_{1}}+\left(\frac{\Sigma_{02}}{S_{02}}\right)^{m_{2}}\right)\right]
$$

This relation is simply a bimodal Weibull model. It perfectly reflects the competition between the two mechanisms. When the fibering orientation is $0^{\circ}$ to the loading axis, the first intensity is much larger than the second and it becomes:

$$
P_{F}=1-\exp \left[-\frac{V_{\Omega}}{V_{0}}\left(\frac{\Sigma_{01}}{S_{01}}\right)^{m_{1}}\right]
$$

When the fibering orientation is $90^{\circ}$ and when the inclusion clusters are large, the second mechanism is predominant and the failure probability is:

$$
P_{F}=1-\exp \left[-\frac{V_{\Omega}}{V_{0}}\left(\frac{\Sigma_{02}}{S_{02}}\right)^{m_{2}}\right]
$$

This simple model can take into account the change of mechanism, the evolution of the fatigue limit and the associated scatter [14.

\section{Conclusions}

Concerning the ability of various experimental techniques to capture the fatigue anisotropy of the forged steel Metasco MC, it can be concluded that:

- The ductility (TE\%) and the fracture toughness, respectively determined by monotonic tensile tests and Charpy tests are both able to detect the 
anisotropic mechanical behavior of the Metasco MC. In both cases, a correlation between the fracture surface and the identified fatigue mechanism is observed: a change in initiation mechanism is the origin of the anisotropic nature.

- The ultimate tensile strength and the yield stress are relatively independent of the orientation of inclusions with respect to the rolling direction.

- For specimens orientated at $0^{\circ}$ relative to the rolled direction, the inclusions are parallel to the applied loading axis and micro-crack initiation is controlled by the material matrix and the prediction of the fatigue strength with the self-heating method is correct. For specimens orientated at $45^{\circ}$ and $90^{\circ}$, elongated manganese sulfide inclusion clusters play a role in crack initiation and the fatigue strength drops significantly. This decreasing of the fatigue strength with the rolling direction is not observed with the selfheating method. It appears that the self-heating method is not capable of capturing the effect of inclusions on the fatigue behavior.

Concerning the self-heating approach, an evolution of the Doudard probabilistic model [18, 19] is proposed. This model considers two different origins of dissipated energy, for each of the observed mechanisms and includes the specific

effect of the inclusion clusters. Experimental work is currently in progress to validate this model.

\section{Acknowledgments}

This work has been performed within the ANR (National Research Agency) Optiforge project, in a partnership including several industrial (Ascometal, Cetim, PSA, Setforge, Transvalor, Ascoforge) and academic (INSA Lyon MATEIS, ENSMP CEMEF, Arts et Métiers ParisTech LAMPA) institutions.

[1] W.A. Spitzig, Metall. Mater. Trans. A 14 (1982) 471-484.

[2] G.R. Speich, W.A. Spitzig, Metall. Mater. Trans. A 13 (1982) 2239-2258.

[3] A.D. Wilson, in: R.J. Glodowski (Eds.), Comparing the effect of inclusions on ductility, toughness, and fatigue properties, ASTM STP 794, Philadelphia, 1983, pp. 130-146.

[4] K. Decamp, L. Bauvineau, J. Besson, A. Pineau, Int. J. Frac. 88 (1997) $1-18$.

[5] D.K Biswas, M. Venkatraman, C.S. Narendranath, U.K. Chatterjee, Metall. Mater. Trans. A 23 (1992) 1479-1492.

[6] S.K. Dhua, A. Ray, S. K. Sen, M.S. Prasad, K.B. Mishra, S. Jha, J. Mater. Eng. Perform. 9 (2000) 700-709.

[7] N. Tsunekage, H. Tsubakino, Mat. Sci. and Tech 18 (2002) 964-972. 
[8] V.P. Raghupathy, V. Srinivasan, H. Krishnan, M.N. Chandrasekharaiah, J. Mater. Sci. 17 (1982) 2112-2126.

[9] N. Cyril, A. Fatemi, B. Crydeman, SAE Int. J. Mater. Manuf. 1 (2009) 218-227.

[10] J. Ma, B. Zhang, D. Xu, E.H. Han, W. Ke, Int. J. Fatigue 32 (2005) 1116-1125.

[11] A.Mateo, L. Llanes, N. Akdut, J. Stolarz, M. Abglada, Int. J. Fatigue 25 (2003) 481-488.

[12] C. Temmel, B. Karlsson, N.G. Ingesten, Metall. Mater. Trans. A 39 (2008) 1132-1144.

[13] G. Lütjering, J. Albrecht, C. Sauer, T. Krull, Mater. Sci. Eng. A (2007) 468-470.

[14] E. Pessard, F. Morel, A. Morel, D. Bellett, Int. J. Fatigue 33 (2011) 568-477.

[15] W.J. Dixon, A.M. Mood, J. Amer. Stat. Assoc. 43 (1948) 109-126.

[16] M.P Luong, Mech. Mater. 28 (1998) 155-163.

[17] F. Cura, G. Curti, R. Sesana, Int. J. Fatigue 27 (2005) 453-459.

[18] C. Doudard, S. Calloch, Eur. J. Mech. A Solids 28 (2009) 233-240.

[19] C. Doudard, S. Calloch, P. Cugy, A. Galtier, F. Hild, Fatigue Fract. Eng. Mater. Struct. 28 (2005) 279-288.

[20] E. Kröner, Acta Metall. 9 (1984) 155-161.

[21] A. Chrysochoos, H. Louche, Int. J. Eng. Sci. 38 (2000) 1759-1788.

[22] A.M. Freudenthal, in: H. Liebowitz (Eds.), Fracture Vol. 2, Academic Press, New York, 1968, pp. 591-619.

[23] M. Abramowitz, A. Stegun, Handbook of Mathematical Functions, Dover, New York, 1965.

[24] J. Ma, B. Zhang, D. Xu, E.H Han, W. Ke, Int. J. Fatigue 32 (2010) 1116-1125.

[25] A. Ekberg, P. Sotkovski, Int. J. Fatigue 23 (2001) 29-34.

[26] C. Peng, Z. Liu, W. Zhu, Eng. Fail. Anal. 18 (2011) 25-35.

[27] G. Baudry, in: C. Bathias, A. Pineau, Hermes (Eds.), Fatigue des Matriaux et des Structures 4, Paris, 2009, pp. 156-161. 
[28] A. Ezanno, C. Doudard, S. Colloch, T.Millot, J.L. Heuz, Procedia Engineering 2 (2010) 967-976.

[29] L. Bodelot, L. Sabatier, E. Charkaluk, P. Dufrenoy, Adv. Eng. Mater. 11 (2009) 723-726. 